\title{
Dynamic-Based Damage Identification using Neural Network Ensembles and Damage Index Method
}

\author{
Ulrike Dackermann*, Jianchun Li, Bijan Samali \\ Centre for Built Infrastructure Research, Faculty of Engineering and Information Technology, \\ University of Technology Sydney, NSW, 2007, Australia
}

\begin{abstract}
This paper presents a vibration-based damage identification method that utilises a "damage fingerprint" of a structure in combination with Principal Component Analysis (PCA) and neural network techniques to identify defects. The Damage Index (DI) method is used to extract unique damage patterns from a damaged beam structure with the undamaged structure as baseline. PCA is applied to reduce the effect of measurement noise and optimise neural network training. PCA-compressed DI values are, then, used as inputs for a hierarchy of neural network ensembles to estimate locations and severities of various damage cases. The developed method is verified by a laboratory structure and numerical simulations in which measurement noise is taken into account with different levels of white Gaussian noise added. The damage identification results obtained from the neural network ensembles show that the presented method is capable of overcoming problems inherent in the conventional DI method. Issues associated with field testing conditions are successfully dealt with for numerical and the experimental simulations. Moreover, it is shown that the neural network ensemble produces results that are more accurate than any of the outcomes of the individual neural networks.
\end{abstract}

\section{Keywords:}

Damage Identification, Artificial Neural Network, Neural Network Ensemble, Structural Health Monitoring, Damage Index Method, Modal Strain Energy, Principal Component Analysis

\footnotetext{
* Corresponding author. E-mail address: udacker@gmail.com; Fax: +61 29514 2633; Tel.: +61 295142651.
} 


\section{Introduction}

During the years following World War II the building industry boomed worldwide. As a result, many civil structures are now, or will soon be, approaching their design lives. Since it is economically not possible to replace all of these aged structures, health monitoring and integrity assessment is necessary to ensure the reliability of the structures and the safety of the public. The dominant procedures for structural condition monitoring today are periodic visual inspections. These, however, are very timeconsuming and costly, and have limited capability to detect damage, especially when the damage occurs inside the structure where it is not visible (Abdel Wahab and De Roeck, 1999). Other non-destructive techniques rely, for instance, on acoustics, ultrasound, magnetic fields, radiography, eddy-currents, thermal fields or X-rays. These methods, however, are so-called local methods and require that the damaged region is known a priori, and the section of the inspected structure is easily accessible (Doebling et al., 1998).

Vibration-based damage identification techniques, in contrast, are global methods and are able to assess the condition of the entire structure simultaneously. These techniques are based on the principle that changes of physical properties in a structure (i.e. stiffness, damping, mass and boundary conditions) reflect damage, which in turn will alter its dynamic characteristics (namely, natural frequencies, mode shapes and modal damping). Vibration-based methods examine changes in the structures dynamic characteristics to detect defects. Eventually, they are related to certain form of pattern recognition problem. Over the past three decades, intensive research has been undertaken in the field of dynamic-based damage identification and many algorithms have been developed. Comprehensive literature reviews on vibration-based damage detection methods were published by Doebling et al. (1996) and Carden and Fanning (2004). Among various vibration-based techniques, especially those using modal parameters, the damage index (DI) method (Stubbs et al., 1992) is particularly promising. This method is based on changes in modal strain energy, and has successfully been applied by many researchers in various fields and applications. Several modifications of the algorithm have been developed and verified by analytical and experimental studies (Choi et al., 2008; Kim and Stubbs, 2002; Stubbs et al., 1995; 
Stubbs and Park, 1996). Farrar and Jauregui (1998a, b) conducted a comparative study of DI method, mode shape curvature method, change in flexibility method, change in uniform load surface curvature method and change in stiffness method. These methods were applied to experimental and numerical modal data of the I-40 bridge in Albuquerque, New Mexico; and the DI method was found to be the one that performed the best in terms of accuracy and reliability. Ndambi, Vantomme and Harri (2002) and Alvandi and Cremona (2006) compared Modal Assurance Criterion (MAC), Coordinate Modal Assurance Criterion (COMAC), flexibility and modal strain energy approaches and concluded that the modal strain energy method was the most precise technique among them and also the most stable one when different levels of noise were induced. Some researchers were even successful in the identification of multiple damage (Patil and Maiti, 2005; Shi et al., 1998). Besides the reported successful applications of the algorithm, the method faces some critical issues. If damage is located close to a node point of a given mode, the defect stays undetected if only this mode is used for detection. In addition, even if multiple modes are used, it is likely to produce false positive damage detection especially when measurements are limited by the number of sensors. Major challenges are faced when the method is applied to real structures under real testing conditions. Errors are encountered due to measurement noise interferences, limited number of sensor arrays or experimental modal analysis uncertainties. The sensitivity to noise especially effects the quantification of defects. Also, the identification of light damage still remains problematic (Barroso and Rodriguez, 2004; Pereyra et al., 1999).

In recent years, the use of Artificial Neural Networks (ANNs) in structural damage detection has gained much attention. ANNs are artificial intelligence that simulate the operation of the human brain. Once trained, they are capable of pattern recognition and classification, and are robust in the presence of noise. These characteristics make ANNs powerful complementary tools in vibrational damage identification. Several researchers employed ANNs in combination with different dynamic-based features for damage assessment. In one of the earliest research papers on ANN based dynamic damage detection, Elkordy, Chang and Lee (1992) demonstrated that using percentage changes in vibrational signatures, rather than using their absolute values, can effectively distinguish between patterns corresponding to different damage states. $\mathrm{Wu}$, Ghaboussi 
and Garrett (1992) were the first to introduce ANN to vibration-based damage detection in civil structures. Their paper investigates the feasibility of ANN in structural damage detection; an experimental three-storey frame structure was excited by base earthquake acceleration and recordings of the Fourier spectrum of the third floor were used as ANN inputs to detect damage. The researchers found that the network was successful in identifying the damage existence for members in the structure and concluded that 'the use of neural networks for structural damage assessment is a promising line of research'. In 2001, Zapico, Worden and Molina presented a damage assessment procedure on a two-storey steel frame and steel-concrete composite floor structure. Three neural network approaches were proposed. For the first and second approaches, the input parameters were the first natural frequency and the first mode shape, respectively; for the third approach, the first two longitudinal bending frequencies were used as inputs. Whereas the first neural network approach failed, in the second approach, the neural network showed an excellent generalisation over the analytical data; however, it failed with experimental data due to the poor accuracy of the extracted mode shapes. The third approach gave reasonable results. The corresponding trained network achieved a good generalization over both the analytical and experimental data. Sahin and Shenoi (2003) used changes in natural frequencies and curvature mode shapes as input features for ANNs for location and severity prediction of numerical and experimental damage in cantilever steel beams. From the network predictions, they reported that the reduction in natural frequency provides the necessary information for the existence and severity of the damage, however, differences in curvature mode shapes severed as a better indicator in the location predictions. Lee and Yun (2006) presented a two step damage identification strategy and demonstrated the method on numerical data and field test data of the old Hannam Grand Bridge in Seoul, Korea. At first, a conventional vibration-based method (DI method) was used to screen potentially damaged members and then, neural networks with a noise injection learning algorithm were trained with mode shape differences between before and after damage to assess the damage. They found that while the conventional method for damage screening produced many false damage alarms, the damage assessment results using neural networks still showed good estimates for all damage cases. Further contributions in this area were made by Xia and Hao (2003) and Bakhary, Hao and Deeks (2007). 
An extension to ANNs is the principle of neural network ensembles, which are a group of networks that are trained independently for the same task and whose outcomes are fused in different stages by ensemble networks. This approach was already used in several field applications and it was observed that the ensemble usually performs better than the best network used alone (Perrone and Cooper, 1993). The idea of multi-stage network training was first employed in the area of vibration-based damage detection by Marwala and Hunt (1999). The researchers applied a two stage neural network ensemble to numerically simulated cantilever beam data, with one network being trained with frequency energies, which are defined as integrals of the real and imaginary components of the frequency response functions over various frequency ranges, and another network trained by using the first five flexural mode shape vectors. The authors found that the ensemble gave a mean error of $7.7 \%$ compared to $9.50 \%$ and $9.75 \%$, respectively, of the individual networks.

Principal Component Analysis (PCA) is a statistical technique for achieving dimensional data reduction and reducing effects of measurement noise. Its application for vibration-based damage detection is reported in several papers (Ni et al., 2006; Trendafilova et al., 2008; Zang and Imregun, 2001b). By projecting data onto the most important principal components, its size can greatly be reduced without significantly affecting the data. Thereby, the effectiveness of neural network training can significantly be improved and unwanted features induced by measurement noise be reduced.

This paper presents a vibration-based damage identification method that utilises the modal strain energy based DI values to analyse dynamic features of beam structures. PCA-compressed damage indicators are used as input patterns for training of backpropagation neural networks. The method is applied to numerical and laboratory beam structures and aims to provide reliable predictions on the location and severity of single damage. Neural network ensembles are utilised in order to take advantage of unique features of individual vibrational mode shapes, such as node point characteristics, varying susceptibility to diverse damage scenarios and different sensitivity to sensor locations. To simulate field-testing conditions, numerically obtained data is polluted with different intensities of white Gaussian noise and issues of limited number of sensor arrays are also incorporated. 


\section{Calculation of damage index}

The conventional DI method, developed by Stubbs, Kim and Topole (1992), utilises an indicator based on relative changes in modal strain energy of a structure before and after damage to detect, locate and quantify defects. Modal strain energy expressed in terms of the derivative of mode shape as the damage index for the $j^{\text {th }}$ element and the $i^{\text {th }}$ mode, $\beta_{i j}$, is given by,

$$
\beta_{i j}=\frac{(E I)_{j}}{(E I)_{j}^{*}}=\frac{\int_{j}\left(\phi_{i}^{\prime \prime *}(x)\right)^{2} d x \int_{0}^{L}\left(\phi_{i}^{\prime \prime}(x)\right)^{2} d x}{\int_{j}\left(\phi_{i}^{\prime \prime}(x)\right)^{2} d x \int_{0}^{L}\left(\phi_{i}^{\prime \prime *}(x)\right)^{2} d x}
$$

where $\phi_{i}{ }^{\prime \prime}$ is the second derivative of mode shape $\phi_{i}$ with respect to $x$ and $L$ is the length of the beam. The asterisk * denotes the damaged case. The derivation of Eqn (1) is discussed in detail in Kim and Stubbs (1995).

To enhance damage detection and produce results related to damage probability, the damage index $\beta_{i j}$ is transformed into the standard normal space and the normalised damage indicator $Z_{i j}$ is given by,

$$
Z_{i j}=\frac{\beta_{i j}-\mu_{\beta i j}}{\sigma_{\beta i j}}
$$

with $\mu_{\beta i j}$ being the mean and $\sigma_{\beta i j}$ the standard deviation of the $\beta_{i j}$ values for all $j$ elements. The estimation of the damage severity for element $j$ is expressed by

$\alpha_{i j}=1-\frac{1}{\beta_{i j}}$

with $\alpha_{i j}$ being the severity estimator. Positive $Z_{i j}$ and $\alpha_{i j}$ values, respectively, indicate the possibility of damage and can therefore be utilised to locate and quantify the defects.

\section{Application of artificial neural networks}


As originally developed for emulating the biology of the human brain, Artificial Neural Networks (ANNs) consist of two primary elements, neurons and weighted interconnections between the neurons. The neurons are linked by transfer functions and arranged in sets of input, hidden and output layer. The strength of the neuron connection is determined by adjusting a variable (weight) and a constant (bias). ANNs can be regarded as nonlinear mathematical functions that map a set of input variables $p_{i}$ $(i=1,2 \ldots d)$ to a set of output variables $a_{k}(k=1,2 \ldots r)$ (Bishop, 1994). Provided enough neurons exist, they are able to represent any function with arbitrary accuracy. Once the networks are trained, they are capable of decision making by means of pattern recognition and classification. They have a fault tolerance and can distinguish between random errors and the desired systematic outputs which make them a robust means for representing model-unknown systems encountered in the real world (Masri et al., 2000). These properties make them particularly attractive in the field of structural damage detection.

A neural network ensemble is a learning paradigm where several neural networks are trained simultaneously for the same task (Sollich and Krogh, 1996). The concept of neural network ensembles (also referred to as committees or classifier ensembles) was first developed by Hansen and Salamon (1990). Hansen and Salamon showed that the generalization ability of a neural network system can significantly be improved through ensembling a number of neural networks and then combining their predictions (Zhou et al., 2002). First, each network in the ensemble is trained individually and then the outputs of each of the networks $a_{e}(e=1,2 \ldots n)$ are fused to produce the ensemble output $a$. A model of a two-stage neural network ensemble is shown in Figure 1.

Figure 1. Model of a two-stage neural network ensemble.

Generally, individual networks can be generated either by varying the design of the networks (i.e. different architecture, transfer functions, training algorithms) or by training the individual networks with different training sets. Many ensembling methods have been proposed in the literature. The most common methods are bagging and boosting. Bagging was proposed by Breiman (1996) and is based on bootstrap sampling (Efron and Tibshirani, 1993). First, several training sets are generated from the original 
training set and then an ensemble neural network is trained from each of those training sets. In boosting, proposed by Schapire (1990), the training sets of the single networks are determined by the performance of former ones. Training variables that are wrongly predicted by previous networks will play more important roles in the training of later networks (Zhou et al., 2002).

\section{Application of Principal Component Analysis (PCA)}

PCA was developed by Pearson (1901) and is one of the most powerful multivariate data analysis techniques for achieving dimensionality reduction. It is a statistical technique that linearly transforms an original set of $k$ variables into a smaller set of $n$ $(n<=k)$ uncorrelated variables, the so-called principal components (PCs). Eigenvalue decomposition of the covariance matrix forms the basis of PCA. The direction of the resulting eigenvectors represents the direction of the PCs, which are weighted according to value of the corresponding eigenvalues. Each PC is a linear combination of the original variables. All the PCs are orthogonal to each other and form an orthogonal basis for the space of the data. The full set of PCs is equal to the original set of the variables. The most significant PCs represent the features that are most dominant in the data set. By removing components that contribute least to the overall variance, the dimension of the original data set can drastically be reduced without significantly affecting the original data (Zang and Imregun, 2001a). Besides the benefit of data reduction, PCA is also a powerful tool for disregarding unwanted measurement noise. As noise has a random feature, which is not correlated with global characteristics of the data set, it is represented by less significant PCs. Therefore, by disregarding PCs of low power, measurement noise is filtered.

Following is a description of the derivation of PCA. Given is the data set $\left[X_{i j}\right]$ with $(i=1,2, \ldots, m)$ and $(j=1,2, \ldots, k)$, where $m$ is the total number of observations (e.g. DI values $Z_{j}$ or $\alpha_{j}$ ) and $k$ the dimension of the observations (e.g. DI data points). First, the mean $\bar{x}_{j}$ and the standard derivation $s_{j}$ of the $j^{\text {th }}$ column are obtained from,

$\bar{x}_{j}=\frac{1}{m} \sum_{i=1}^{m} x_{i j}$

and 
$s_{j}=\sqrt{\frac{\sum_{i=1}^{m}\left(x_{i j}-\bar{x}_{j}\right)^{2}}{m}}$

Then, the data set $[X]$ is transformed into the standard normal space yielding the variation matrix $[\tilde{X}]$. A normalised element $\tilde{x}_{i j}$ is given by,

$\tilde{x}_{i j}=\frac{x_{i j}-\bar{x}_{j}}{s_{j}}$

The covariance matrix $[C]$ is expressed as,

$[C]=\frac{[\tilde{X}]^{T}[\tilde{X}]}{m-1}$

Finally, the PCs are obtained from,

$[C]\left\{P_{i}\right\}=\lambda_{i}\left\{P_{i}\right\}$

which is the eigenvalue decomposition of the covariance matrix $[C]$, with $\lambda_{i}$ being the $i^{\text {th }}$ eigenvalue and $\left\{P_{i}\right\}$ the corresponding eigenvector. The first PC, which is the largest eigenvalue and its associated eigenvector, represents the direction and amount of maximum variability in the original data set. The second PC, which is orthogonal to the first PC, represents the second most significant contribution from the data set, and so on (Fang and Tang, 2005).

\section{Methodology}

This paper presents a vibration-based damage identification method that determines the location and the severity of single defects in numerical and experimental beam structures. Damage is identified by artificial neural networks, which utilise PCAcompressed DI values as input patterns. To simulate field-testing conditions, numerically obtained data is polluted with different intensities of white Gaussian noise and issues of limited number of sensor arrays are also incorporated. To disregard unwanted features introduced by noise, PCA is applied to the damage indicators and only the most significant PCs are utilised for neural network training. Instead of using single neural networks, an approach based on neural network ensembles is used in order 
to take advantage of unique features of individual vibrational mode shapes, such as node point characteristics, varying susceptibility to diverse damage scenarios and different sensitivity to sensor locations and mode shape interpolation. In the neural network ensemble, PCA-compressed DI values of each individual vibrational mode are first evaluated in individual neural networks and then the individual network outcomes are fused in the ensemble.

Firstly, modal parameters are to be extracted from time history data of the numerical and the laboratory beams by means of experimental modal analysis procedures. Therefore, modal testing is conducted for the experimental beams, and transient analysis with subsequent noise pollution is performed for the numerical structures in order to obtain the response time histories. Real testing conditions regarding coarse sensor arrays are incorporated by using a minimal number of measurement points for the higher mode shapes to be considered. To improve the damage detection results, cubic spline interpolation techniques are adopted to reconstruct finer mode shapes. Secondly, from the identified mode shapes the modal strain energy based DI values $Z_{j}$ and $\alpha_{j}$ are derived. Thirdly, to disregard unwanted features such as those caused by measurement noise, the DI values are transferred to the principal component space and only the most dominant components of the data are selected for subsequent neural network training. Fourthly, sets of individual neural networks are trained to map PCA-compressed DI values from individual vibrational modes to the location and the severity of damage. Finally, a neural network ensemble fuses the outcomes of the individual networks and a conclusive overall damage prediction is obtained.

The method is verified by two models. The first model is based on numerical simulations, in which response time history data are polluted with white Gaussian noise; and the second model is experimental, simulating a real test.

\section{Damage identification procedure}

\subsection{Numerical model}

A numerical model of a steel beam with the dimensions of $12 \mathrm{~mm}$ by $32 \mathrm{~mm}$ by $2,400 \mathrm{~mm}$ is created using the finite element analysis package ANSYS (2005a). The 
element type used is SOLID45, which is a three dimensional structural solid defined by eight nodes having translations in the nodal $x, y$ and $z$ directions. The cross-section of the beam is modelled with 4 elements across the height and 4 elements along the width. A division into 201 nodes and 200 elements in the longitudinal direction of the model is chosen in accordance with previous sensitivity studies undertaken by Choi et al. (2007). The beam model is of steel with modulus of elasticity of $200,000 \mathrm{~N} / \mathrm{mm}^{2}$. The support conditions are set as pin-pin. A schematic model of the numerical beam is shown in Figure 2 (a).

Figure 2. Finite element modelling of (a) pin-pin supported steel beam and (b) light size damage with a width of $1 \mathrm{~mm}$ and a height of $4 \mathrm{~mm}$.

Four different damage locations are considered, which are at locations $4 / 8^{\text {th }}, 5 / 8^{\text {th }}$, $6 / 8^{\text {th }}$ and $7 / 8^{\text {th }}$ of the span length. The locations are denoted as ' 4 ', ' 5 ', ' 6 ' and ' 7 ', and are shown in Figure 2 (a). For each of these locations four different damage severities, termed as extra light ('XL'), light ('L'), medium ('M') and severe (' $\mathrm{S}$ '), are investigated. All inflicted damage are notch type, $1 \mathrm{~mm}$ in length and $1 \mathrm{~mm}, 4 \mathrm{~mm}, 8 \mathrm{~mm}$ and $12 \mathrm{~mm}$ in height. This corresponds to a cross-section loss of the second moment of area, I, of $9.09 \%, 33.01 \%, 57.81 \%$ and $75.59 \%$, respectively. Damage is modelled by rectangular openings from the soffit of the beam along the span length. The mesh density is refined in the vicinity of the defect as displayed in Figure 2 (b). In total, 16 different damage cases are generated.

To obtain response time history data of the numerical beam models, transient analysis is performed using ANSYS. A force of $800 \mathrm{~N}$, which is a typical impact force observed from experimental hammer excitation, is applied at a reference point (here at location ' 5 ') and the response time histories of the beam are recorded at nine equally spaced points. These nine points represent measurement sensors in real testing. The following sentences were modified and added: In order to consider noise, which is present in a real test, white Gaussian noise of four intensities ( $1 \%, 2 \%, 5 \%$ and $10 \%)$ is added to the excitation signal and the response time histories. The Matlab function 'awgn' with the noise-to-signal ratio function $20 \log _{10}(r)$ is therefore used. For the noise intensities of $1 \%, 2 \%, 5 \%$ and $10 \%, \mathrm{r}$ is set to $0.01,0.02,0.05$ and 0.1 , respectively, 
and white Gaussian is randomly added to the original recorded data. For each level of noise, three different sets of noise-polluted data are generated. The different sets of time history data are transformed into the frequency spectra using Fast Fourier Transform (FFT), which is given by,

$$
x(k)=\sum_{j=0}^{N-1} X(j) W_{N}^{-j k}
$$

In the above equation, $x(k)$ represents the discrete series at the time instant $k$ of a sampled data $N(k=0,1 \ldots N-1$ and $j=0,1 \ldots N-1)$, where $W_{N}$ equals $e^{-i 2 \pi / N}$. The Frequency Response Function (FRF) is estimated by dividing cross-spectra between input and output with auto-spectra of input. The modal parameters of the first seven flexural modes are identified by performing experimental modal analysis procedures utilising the software from LMS (LMS CADA-X).

To enhance the quality and effectiveness of the damage identification, the obtained mode shape vectors are reconstructed from 9 to 41 data points utilising cubic spline interpolation techniques by using the Spline function in Matlab. In the operation, a tridiagonal linear system is solved to describe the coefficients of various cubic polynomials, which make up the interpolating spline. A detailed description on the reconstruction of mode shapes using cubic spline data interpolation can be found in Choi et al. (2006). By correlating modal strain energy which is a function of the refined mode shape curvature vectors of the undamaged beam to those of the different damaged beams, the DI values $Z_{j}$ and $\alpha_{j}$ are determined following the procedure described in section 2. For each noise pollution level and each individual mode, a total of $144 \mathrm{Z}_{\mathrm{j}}$ and $\alpha_{\mathrm{j}}$ damage indices, respectively, are generated by relating each noise-polluted undamaged case to each of the noise-polluted damaged cases (4 damage locations $\times 4$ damage severities $\times 3$ noise-polluted undamaged data sets $\times 3$ noise-polluted damaged data sets).

\subsection{Experimental model}

Laboratory testing of four pin-pin supported steel beams were undertaken in the structures laboratory of the University of Technology Sydney (UTS). The dimensions of 
the beams were $12 \mathrm{~mm}$ by $32 \mathrm{~mm}$ by $2,400 \mathrm{~mm}$, which are the same as the dimensions of the numerical models. A picture of the experimental set up is displayed in Figure 3 (a).

Figure 3. (a) Laboratory test set up and (b) experimental saw cut damage of light size.

Each of the steel beams was inflicted with four different severities of single damage situated at locations $4 / 8^{\text {th }}, 5 / 8^{\text {th }}, 6 / 8^{\text {th }}$ and $7 / 8^{\text {th }}$ of the span length. The four damage severities of extra light, light, medium and severe, were again $1 \mathrm{~mm}$ in length and $1 \mathrm{~mm}$, $4 \mathrm{~mm}, 8 \mathrm{~mm}$ and $12 \mathrm{~mm}$, respectively, in height. The damage was introduced by saw cuts from the soffit of the beam. The damage of light severity is depicted in Figure 3 (b).

The modal parameters of the beams are obtained by performing experimental modal testing and analysis. In modal testing, the beams are excited by a modally tuned impact hammer. Nine equally spaced piezoelectric accelerometers, mounted on the top surface of the beams, were used to measure the beam response. The signals of the hammer and the accelerometers were first amplified by signal conditioners and then recorded by a data acquisition system. The sampling rate was set to $10,000 \mathrm{~Hz}$ for a frequency range of 5,000 $\mathrm{Hz}$ and 8,192 data points, thus giving a frequency resolution of $0.61 \mathrm{~Hz}$ per data point. The main data acquisition system consists of a Hewlett Packard state-of-theart VXI system equipped with LMS CADA-X. The acquired time history data were then transformed into the frequency domain and by performing modal analysis, following the same procedures described in section 6.1, the modal parameters are determined. The experimental modal testing set up and modal analysis procedures are shown in Figure 4.

Figure 4. Schematic diagram of experimental modal testing and analysis.

The identified first seven flexural mode shapes are again reconstructed from 9 to 41 data points and the DI values $Z_{j}$ and $\alpha_{j}$ are derived. As each damage case and the undamaged state is tested 5 times, a total of $400 \mathrm{Z}_{\mathrm{j}}$ and $\alpha_{\mathrm{j}}$ damage indices are generated 
for each mode (4 damage locations x 4 damage severities x 5 undamaged data sets $\mathrm{x}$ 5 damaged data sets).

\subsection{Principal Component Selection}

PCA is applied to the damage indicators in order to extract the most dominant characteristics of the data and thereby to disregard unwanted features introduced by noise. The 'princomp' function in MATLAB is utilised to transfer the DI values to the principal component space based on the equations of section 4 . The DI values of the numerical and the experimental beams are arranged in matrices of $m \times k$, where $m$ are the observations (144 and 400 DI values, respectively) and $k$ the dimension of the observations (41 DI data points). After the projection, each of the observations is presented by 41 principal components. The cumulative contribution percentages of all $41 \mathrm{PCs}$ of $\mathrm{Z}_{\mathrm{j}}$ indices of mode 1 of numerical noise-polluted beam data are shown in Figure 5.

Figure 5. Cumulative contribution of PCs obtained from $Z_{j}$ derived from mode 1 of numerical data.

From the graph, it can be seen that the first component accounts for $35.5 \%$ of the original data. The first and the second PCs together contribute to $60.1 \%$ of the data and the summation of the first three PCs represents $79.8 \%$ of the original data. The cumulative contribution from the $11^{\text {th }}$ to the $41^{\text {st }} \mathrm{PC}$ is less than $1 \%$. Therefore, the first ten PCs, which represent $99.1 \%$ of the original data, are regarded as most significant components and used as input parameters for the neural networks. A very similar contribution distribution is obtained from the damage indices $Z_{j}$ and $\alpha_{j}$ of the experimental simulations and hence the first ten PCs are also chosen as input features.

\subsection{Artificial neural network model}

Ensembles of supervised feed-forward multi-layer neural networks are created to identify damage. The ten most dominant PCs of the damage indices $Z_{j}$ and $\alpha_{j}$ are utilised, respectively, to train neural networks to estimate the location and the severity 
of damage. First, individual neural networks are trained with PCs that are separated by vibrational modes (each individual network evaluates PCA-compressed DI values derived from one of the seven captured modes). Then, the outcomes of the individual neural networks are combined in a neural network ensemble and a final damage prediction is obtained. The individual neural networks comprise of one input layer with 10 nodes, representing the first ten PCs of the damage indices $Z_{j}$ and $\alpha_{j}$, respectively; four hidden layers with 8, 6, 4 and 2 nodes; and one single node output layer estimating the location (in length along the beam) or the severity (in loss of the second moment of area, I) of the damage. The number of nodes of each hidden layer was determined following the 'geometric pyramid' rule as described in Masters (1993). The network ensemble is designed with seven input nodes, which are the outputs of the seven individual mode networks; three hidden layers of 7, 5, and 3 nodes; and one output node estimating the damage location or severity. The transfer functions used are hyperbolic tangent sigmoid functions. This transfer function is chosen, as it produces more accurate results and faster training times when compared to any of the other available transfer functions, which are linear and logistic functions. Training is performed utilising the back-propagation conjugate gradient descent algorithm. The input data is divided into three sets; a training, a validation and a testing set. While the network adjusts its weight from the training samples, its performance is supervised utilising the validation set to avoid overfitting. The network training stops when the error of the validation set increases while the error of the training set still decreases, which is the point when the generalisation ability of the network is lost and overfitting occurs. The division of the available input samples into the three sets (training, validation and testing) is conducted according to a partitioning system termed chessboard selection. For the laboratory data, the chessboard selection principle is illustrated in Table 1. To calculate the damage indices $Z_{i}$ and $\alpha_{i}$, a set of undamaged data is correlated to a set of damaged data, as described above. As each undamaged and damaged state was tested five times, a total of 25 data sets are obtained for each damage case. To select a diverse range of data for each set ('train', 'val' and 'test'), data along a diagonal line of the input samples are selected for each set (as illustrated in Table 1). For the laboratory data, each damage case is divided into sets of 15 samples for training, 5 samples for validation and 5 samples for testing. Thereby, for the entire data set of 400 laboratory samples, 240 are 
allocated for training, and 80 each for validation and testing. For the 144 samples of the noise-polluted numerical data, 82 are allocated for training and 31 each for validation and testing. The design and operation of all neural networks is performed with the software Alyuda NeuroIntelligence version 2.2 from Alyuda Research Inc.

Table 1. Chessboard selection for laboratory beam data.

\section{Results and discussion}

\subsection{Damage index values}

The DI values $Z_{j}$ and $\alpha_{j}$, which give indications on the location and the extent of damage, are the first intermediate results obtained from the developed procedure. As an example, some damage indicators $Z_{j}$ and $\alpha_{j}$ of numerical noise-free beam simulations are shown in Figure 6 . In the figures, the $\mathrm{x}$-axis shows the length of the beam with ' 1 ' to ' 7 ' indicating 7 possible damage locations and the y-axis the damage index. As only positive DI values indicate damage, all negative numbers are set to zero. The actual damage location is marked with a straight line. Figure 6 (a) shows the damage indicator $\mathrm{Z}_{\mathrm{j}}$ of a beam with a defect at locations ' 5 '. Here a clear indication of the damage location can be seen. The illustrations of Figure 6 (b) and (c) depict the severity estimator $\alpha_{j}$ of beams, which are damaged at location ' 4 ' with the damage severities of medium and severe. The different magnitudes of the severity estimator $\alpha_{j}$ clearly indicate the different extents of the defects.

Figure 6. Damage indicators of noise-free numerical simulations derived from mode 1 . (a) $Z_{j}$ of a damage situated at location ' 5 '. (b) and (c) $\alpha_{\mathrm{j}}$ of a damage situated at location ' 4 ' of medium and severe severity, respectively.

From the derived DI values, a couple of issues associated with the damage index method itself and real life testing limitations of coarse sensor nets and noise interferences are identified. Firstly, if damage is located at a node point of a mode shape it cannot be detected. For DI value $Z_{j}$, even false damage indications occur, as presented in Figure 7 (a), which shows damage indicator $Z_{j}$ derived from mode 4 of a noise-free 
numerical beam damaged at mid-span. Secondly, if only a limited number of measurement data is available, as is the case in real applications, false positive damage indications occur in a couple of damage cases. This phenomenon is shown in Figure 7 (b), which displays $Z_{j}$ derived from mode 3 of a noise-free numerical beam damaged at location ' 5 '. Here, besides the correct damage location, a false indication at location ' 6 ' is visible. Thirdly, damage that is located close to the supports is slightly misallocated for all cases. This is presented in Figure 7 (c) for $Z_{j}$ derived from mode 1 of a noise-free numerical beam damaged at location ' 7 '. When the damage index method is used alone to detect damage, defects may falsely be identified. However, the faulty indications by the damage index method are recurring patterns. By utilising neural network techniques, with their ability to recognise patterns, the damage identification process can be improved and critical issues overcome.

Figure 7. $Z_{j}$ values of noise-free numerical simulations derived from (a) mode 4 of a beam damaged at location ' 4 ', (b) mode 3 of a beam damaged at location ' 5 ' and (c) mode 1 of a beam damaged at location ' 7 '.

Further, the damage indices are very sensitive to noise interferences and modal analysis uncertainties. This phenomenon can be seen in Figure 8, which displays damage indicator $\mathrm{Z}_{\mathrm{j}}$ of numerical simulations polluted with three different noise signals, all of $2 \%$ white Gaussian noise. Here damage is present at location ' 5 '. Whereas the derived DI value of Figure 8 (a) gives the correct damage location, the indices of Figure 8 (b) and Figure 8 (c) either show an additional damage or misallocate the defect. By transferring the damage indicators into the principal component space and considering only the most significant PCs, only the main characteristics of the data are considered and thereby, uncorrelated features, introduced by noise, are disregarded.

Figure 8. $Z_{j}$ values of numerical simulations polluted with three different signals of $2 \%$ white Gaussian noise derived from mode 1 of a beam damaged at location ' 5 '.

\subsection{Individual neural network outcomes}


Individual neural networks are trained with PCA-compressed damage indices to identify defects. The PCs of damage indicator $Z_{j}$ are utilised to determine the location of the damage and the PCs of the severity estimator $\alpha_{\mathrm{j}}$ are used to quantify the damage extent. In the following sections, the outcomes of the individual networks, trained to identify locations and severities of noise-polluted numerical and experimental steel beams, are presented. In the subsequent figures, the $\mathrm{x}$-axis displays the damage cases sorted by their locations $\left(\mathrm{L}_{4}, \mathrm{~L}_{5}, \mathrm{~L}_{6}\right.$ and $\left.\mathrm{L}_{7}\right)$ and their severities $\left(\mathrm{S}_{\mathrm{XL}}, \mathrm{S}_{\mathrm{L}}, \mathrm{S}_{\mathrm{M}}\right.$ and $\left.\mathrm{S}_{\mathrm{S}}\right)$. The $y$-axis represents the normalised error $E_{\text {norm }}$ of either the localisation or the quantification outcomes. The normalised error is defined as

$$
E_{\text {norm }}(d)=\frac{\left(T_{d}-O_{d}\right)}{L_{\max }}
$$

and

$$
E_{\text {norm }}(d)=\frac{\left(T_{d}-O_{d}\right)}{S_{\max }}
$$

respectively, where $d$ is the damage case, $T_{d}$ the target value of $d, O_{d}$ the network output value of $d, L_{\max }$ the total length of the beam (here $2.4 \mathrm{~m}$ ) and $S_{\max }$ the maximum severity of a damage (here $100 \%$ loss of the second moment of area, I). A marked band around the $0 \%$ error axis symbolises the area in which the network estimations must fall in order to correctly categorise the damage. For the localisation of damage, the band ranges from $-6.25 \%$ to $+6.25 \%$ normalised error, representing the mid points inbetween two damage locations $\left(E_{\text {norm }}(\operatorname{mid}\right.$ point $\left.)= \pm 0.15 \mathrm{~m} / 2.4 \mathrm{~m}= \pm 6.25 \%\right)$. The band of the damage quantifications ranges from $-12 \%$ to $+12 \%$ normalised error, representing the average mid points in-between two severity levels. The network performances of the training, validation and testing sets are presented below in absolute mean of the normalised error $E_{\text {norm }}$ abbreviated as AMNE.

\subsubsection{Individual neural network outcomes of numerical model}

Individual neural networks trained with noise-polluted numerical data give outcomes that vary significantly in their accuracies. Depending on the mode from which the DI values were derived, the network results reflect the different characteristics of the individual modes. Also, the noise pollution level has an effect on the outcomes of the 
neural networks. The individual neural network performances of the training, validation and testing set of modes 1 to 7 are presented in Table 2 in absolute mean of normalised error (AMNE).

Table 2. Neural network performances (in absolute mean of normalised error (AMNE)) of noise-polluted numerical beam simulations to identify damage locations and severities.

As examples, Figure 9 (a) to (f) display the testing set outcomes of networks trained with the first 10 PCs of DI value $Z_{j}$ derived from mode 1 , mode 4 and mode 5 , respectively, that are polluted with $1 \%$ or $10 \%$ white Gaussian noise. From the figures it can be seen that many damage cases are incorrectly located for both networks of mode 1 . This phenomenon can be explained by the small curvature value of mode 1 , which results in a low sensitivity to damage. Misidentifications of the networks of mode 4 occur almost exclusively at the node points of mode 4 , which are locations ' 4 ' and ' 6 '. Damage cases from locations ' 5 ' and ' 7 ' are all correctly identified for the mode 4 network trained with $1 \%$ noise polluted data and only two extra-light damage cases are wrongly located for data of noise pollution level $10 \%$. The network predictions of mode 5 traine $\mathrm{d}$ with $1 \%$ noise polluted data are correct for all but three extra-light damage cases; a noise pollution level of $10 \%$ still gives precise damage locations for all medium and severe defects. From these outcomes it can be observed that individual characteristics of the different modes have a major influence on the neural network outcomes. This highlights how important it is to separate the DI values by modes. The intensity of noise in contrast seemed to have a lesser effect on the damage identification results. This shows the effectiveness of the noise filtering capacity of PCA and neural networks. Note: The results of the individual networks trained to estimate the severity of the defects show very similar outcome characteristics to the networks trained to identify the damage locations.
(a) mode 1 network $-1 \%$ noise
(b) mode 1 network - $10 \%$ noise
(c) mode 4 network $-1 \%$ noise
(d) mode 4 network - $10 \%$ noise
(e) mode 5 network $-1 \%$ noise
(f) mode 5 network - $10 \%$ noise

Figure 9. Testing set outcomes of individual neural networks trained with PCA-compressed $Z_{j}$ values from numerical simulations polluted with $1 \%$ and $10 \%$ white Gaussian noise, respectively, derived from (a) and (b) mode 1, (c) and (d) mode 4, and (e) and (f) mode 5. 


\subsubsection{Individual neural network outcomes of the experimental model}

The outcomes of the individual networks trained with experimental beam data are quite different to those trained with numerically generated data. The performances of the training, validation and testing set of the individual networks of modes 1 to 7 are listed in Table 3.

Table 3. Neural network performances (in absolute mean of normalised error (AMNE)) of experimental beams to identify damage locations and severities.

For the experimental beams, damage localisation is successful for all damage cases of the networks trained with PCs of $Z_{j}$ values derived from mode 1 , mode 2 , mode 3 and mode 4, as displayed in Figure 10 (a) for the network of mode 2. The testing set outcomes of the network of mode 5 show seven wrongly located damage cases (out of the 80 testing samples); mode 6 and mode 7 networks have false localisations for 13 and 19 damage cases, respectively. For the localisation of experimental damage cases, issues associated with damage cases that are situated at node points of mode 2 and mode 4 seem to have been overcome. (All damage cases at the node points of mode 2 and mode 4 are correctly identified.) This phenomenon can be explained by the fact that the experimental beam set up is not perfect. The beam supports may not have ideal pin-pin conditions, and the damage locations may not be situated at the exact division points. These imperfections may thereby avoid the singularities at the node points and associated damage identification issues are hence overcome. The false identifications of the networks of mode 5, mode 6 and mode 7 are due to difficulties faced with the determination of these higher modes during the process of experimental modal analysis. The individual networks trained to identify the quantities of the experimental damage cases, correctly identify all severe defects. Many extra-light, light and medium damage cases, however, are falsely quantified by all seven individual mode networks, as shown for the network of mode 2 in Figure 10 (b). The wrongly identified damage cases are not consistent across the individual networks, i.e. each individual mode network falsely quantifies different damage cases, which shows the complexity of dealing with experimental data. 
(a) damage localisation - mode 2 network

(b) damage quantification- mode 2 network

Figure 10. Training set outcomes of individual neural networks trained with PCs of DI values derived from mode 5 of experimental beam data to (a) locate and (b) quantify damage.

\subsection{Neural network ensemble outcomes}

To determine the damage characteristics based only on the outcomes of the individual networks is not reliable as results of damage estimations differ a lot depending on individual mode characteristics, damage locations and severities, as shown in the previous two sections. To obtain reliable damage identification, a conclusive, intelligent fusion of the network outcomes is necessary. This is achieved by a neural network ensemble, which combines the outcomes of the individual networks. For the network ensembles trained with numerical data, the damage localisation outcomes of the networks trained with different noise pollution levels are presented in Figure 11. From the figures, it can be observed that for a noise pollution level of $1 \%$ and $2 \%$ only one or two extra-light damage cases are wrongly identified. The networks trained with data of $5 \%$ and $10 \%$ noise intensity precisely locate all medium and severe defects. For the quantification of numerically simulated damage cases, the network ensembles of $1 \%, 2 \%$ and $5 \%$ noise polluted data give correct identification of all defects. The network ensemble trained with $10 \%$ noise polluted data falsely quantifies one extra-light and two light damage cases. For the experimental beams, the localisation network ensemble precisely identifies all damage cases. The network ensemble that aims to determine the damage extent correctly quantifies all light, medium and severe damage cases; five extra-light defects are falsely identified. These results clearly show the effectiveness of the neural network ensemble. Furthermore, it was observed that the ensemble outcomes are more accurate than any of the outcomes of the individual neural networks.
(a) network ensemble $-1 \%$ noise
(b) network ensemble $-2 \%$ noise
(c) network ensemble $-5 \%$ noise
(d) network ensemble $-10 \%$ noise

Figure 11 Outcomes if neural network ensemble trained with numerical data of (a) $1 \%$, (b) $2 \%$, (c) $5 \%$ and (d) $10 \%$ noise pollution to estimate the location of damage. 
(a) network ensemble - experimental data for damage localisation (b) network ensemble - experimental data for damage severity estimation

Figure 12. Outcomes of neural network ensemble trained with experimental data to estimate (a) the location and (b) the severity of damage.

\section{Conclusions}

This paper presents a vibration-based damage identification method that utilises the advantage of damage index method in combination with PCA and neural network techniques to identify location and severity of single damage. With the use of ANN with PCA, problems of the conventional DI method are overcome and real-life testing issues associated with limited number of sensor arrays, measurement noise and incomplete data sets are addressed. By transferring DI values into the principal component space and disregarding PCs of low power, the effects of noise are further reduced and neural network training optimised. The neural network ensemble approach is utilised in order to intelligently fuse outcomes of individual NNs for an optimised solution. The individual neural networks take advantage of distinct features of separated DI values in each individual mode shape for better pattern recognition. To simulate field-testing conditions, different intensities of white Gaussian noise are added to numerical data. The developed method is verified by two models. The first model is based on numerical simulations, which are polluted with white Gaussian noise; and the second model is experimental, simulating a real test. The results of the individual networks show that the individual characteristics of the different modes of the damage indices have a major influence on the network outcomes, which highlights the importance to separate the DI values by modes before the network training. The network outcomes also demonstrate the effectiveness of the noise filtering capacity of PCA and neural networks. The final damage predictions of the neural network ensembles are found to give results that are more accurate than any of the outcomes of the individual neural networks; and it is shown that the presented damage identification approach is effective and reliable in dealing with issues of real life testing. 


\section{Acknowledgements}

The authors wish to thank the Centre for Built Infrastructure Research (CBIR), Faculty of Engineering and Information Technology (FEIT), University of Technology Sydney (UTS) for supporting this project. Within the FEIT, the authors wish to express their gratitude to the staff of UTS Structures Laboratory for their assistance in conducting the experimental works. Alyuda Research Inc. is gratefully acknowledged for providing a free copy of their Alyuda NeuroIntelligence software.

\section{References}

Abdel Wahab, M. M. and De Roeck, G. (1999). "Damage detection in bridges using modal curvatures: Application to a real damage scenario", Journal of Sound and Vibration, Vol. 226, No. 2, pp 217-235.

Alvandi, A. and Cremona, C. (2006). "Assessment of vibration-based damage identification techniques", Journal of Sound and Vibration, Vol. 292, No. 1-2, pp 179-202.

Bakhary, N., Hao, H. and Deeks, A., (2007). "Damage detection using artificial neutral network with consideration of uncertainties", Engineering Structures, Vol. 29, No. 11, pp 28062815.

Barroso, L. R. and Rodriguez, R. (2004). "Damage detection utilizing the damage index method to a benchmark structure", Journal of Engineering Mechanics, Vol. 130, No. 2, pp 142-151.

Bishop, C. M. (1994). "Neural networks and their applications", Review of Scientific Instruments, Vol. 65, No. 6, pp 1803-1832.

Breiman, L. (1996). "Bagging predictors", Machine Learning, Vol. 24, No. 2, pp 123 - 140.

Carden, E. P. and Fanning, P. (2004). "Vibration based condition monitoring: A review", Structural Health Monitoring, Vol. 3, No. 4, pp 355-377.

Choi, F. C. (2007). Assessment of the Structural Integrity of Bridges using Dynamic Approaches, $\mathrm{PhD}$ Thesis, Faculty of Engineering, University of Technology Sydney, Australia.

Choi, F. C., Li, J., Samali, B. and Crews, K. (2008). "Application of the modified damage index method to timber beams", Engineering Structures, Vol. 30, No. 4, pp 1124-1145.

Choi, F.C., Li, J., Samali, B. and Crews, K. (2006). "Impact of different numerical techniques on damage identification in structures", Proceedings of the Tenth East Asia-Pacific Conference on Structural Engineering and Construction. Bangkok, Thailand, pp 111-116. 
Doebling, S.W., Farrar, C. R., Prime, M.B. and Shevitz, D.W. (1996). "Damage identification and health monitoring of structural and mechanical systems from changes in their vibration characteristics: A literature review", Los Alamos National Laboratory Report, LA-13070MS.

Doebling, S.W., Farrar, C.R. and Prime, M.B. (1998). "Summary review of vibration-based damage identification methods", Shock and Vibration Digest, Vol. 30, No. 2, pp 91-105.

Efron, B. and Tibshirani, R. (1993). An Introduction to the Bootstrap, Chapman and Hall, New York.

Elkordy, M., Chang, K.C. and Lee, G.C. (1992). "Application of neural network classifier in vibrational signature analysis", Proceedings of the 8th Annual Conference of Computation in Civil Engineering, ASCE, pp. 1066-1073.

Fang, X. and Tang ,J. (2005). "Frequency response based damage detection using principal component analysis", Proceedings of the IEEE International Conference on Information Acquisition. Hong Kong and Macau, China, pp 407-412.

Farrar, C.R. and Jauregui, D.A. (1998a). "Comparative study of damage identification algorithms applied to a bridge: I. Experiment", Smart Materials and Structures, Vol. 7, No. 5, pp 704-719.

Farrar, C.R. and Jauregui, D.A. (1998b). "Comparative study of damage identification algorithms applied to a bridge: II. Numerical study", Smart Materials and Structures, Vol. 7, No. 5, pp 720-731.

Hansen, L.K. and Salamon, P. (1990). "Neural network ensembles", Proceedings of the IEEE Transactions on Pattern Analysis and Machine Intelligence, Vol. 12, No. 10, pp 993 - 1001.

Kim, J. T. and Stubbs, N. (2002). "Improved damage identification method based on modal information", Journal of Sound and Vibration, Vol. 252, No. 2, pp 223-238.

Kim, J.T. and Stubbs, N. (1995). "Model-uncertainty impact and damage detection accuracy in plate girder", Journal of Structural Engineering, Vol. 121, No. 10, pp 1409-1417.

Lee, J.J. and Yun, C.B. (2006). "Damage diagnosis of steel girder bridges using ambient vibration data", Engineering Structures, Vol. 28, No. 6, pp 912-925.

Marwala, T. and Hunt, H.E.M. (1999). "Fault identification using finite element models and neural networks", Mechanical Systems and Signal Processing, Vol. 13, pp 475-490.

Masri, S.F., Smyth, A.W., Chassiakos, A.G., Caughey T.K. and Hunter, N.F. (2000). "Application of neural networks for detection of changes in nonlinear systems", Journal of Engineering Mechanics, Vol. 126, No. 7, pp 666-676.

Masters, T. (1993). Practical Neural Network Recipes in C++, Academic Press, London. 
Ndambi, J.M., Vantomme, J. and Harri, K. (2002). "Damage assessment in reinforced concrete beams using eigenfrequencies and mode shape derivatives", Engineering Structures, Vol. 24, No. 4, pp 501-515.

Ni, Y.Q., Zhou, X.T. and Ko, J.M. (2006). "Experimental investigation of seismic damage identification using PCA-compressed frequency response functions and neural networks", Journal of Sound and Vibration, Vol. 290, No. 1-2, pp 242-263.

Patil, D. P. and Maiti, S. K. (2005). "Experimental verification of a method of detection of multiple cracks in beams based on frequency measurements", Journal of Sound and Vibration, Vol. 281, No. 1-2, pp 439-451.

Pearson, K. (1901). "On lines and planes of closest fit to systems of points in space", Philosophical Magazine, Vol. 2, No. 6, pp 559-572.

Pereyra, L.R., Osegueda, R.A., Carrasco, C.J. and Ferregut, C.M. (1999). "Damage detection in a stiffened plate using modal strain energy differences", Proceedings of the SPIE Nondestructive Evaluation of Aging Aircraft, Airports and Aerospace Hardware III, pp 211-222.

Perrone, M.P. and Cooper, L.N. (1993). "When networks disagree: Ensemble methods for hybrid neural networks", Artificial Neural Networks for Speech and Vision, Chapman and Hall, London, pp 126-142.

Sahin, M. and Shenoi, R.A. (2003). "Quantification and localisation of damage in beam-like structures by using artificial neural networks with experimental validation", Engineering Structures, Vol. 25, No. 14, pp 1785-1802.

Schapire, R.E. (1990). "The strength of weak learnability", Machine Learning, Vol. 5, No. 2, pp $197-227$.

Shi, Z.Y., Law, S.S. and Zhang, L.M. (1998). "Structural damage localization from strain energy change", Journal of Sound and Vibration, Vol. 218, No. 5, pp 825-844.

Sollich, P. and Krogh, A. (1996). "Learning with ensembles: How overfitting can be useful", Advances in Neural Information Processing Systems 8, MIT Press, Denver, pp 190-196.

Stubbs, N., Kim, J.T. and Farrar, C.R. (1995). "Field verification of a nondestructive damage localization and severity estimation algorithm", Proceedings of the 13th International Modal Analysis Conference: SPIE, pp 210-218.

Stubbs, N., Kim, J.T. and Topole, K. (1992). "An efficient and robust algorithm for damage localization in offshore platforms", Proceedings of the ASCE Tenth Structures Congress, Antonio, Texas, USA, pp 543-546.

Stubbs, N. and Park, S. (1996). "Optimal sensor placement for mode shapes via Shannon's sampling theorem", Microcomputers in Civil Engineering, Vol. 11, No. 6, pp 411-419. 
Trendafilova, I., Cartmell, M.P. and Ostachowicz, W. (2008). "Vibration-based damage detection in an aircraft wing scaled model using principal component analysis and pattern recognition", Journal of Sound and Vibration, Vol. 313, No. 3-5, pp 560-566.

Wu, X., Ghaboussi, J. and Garrett Jr., J.H. (1992). "Use of neural networks in detection of structural damage", Computers and Structures, Vol. 42, No. 4, pp 649-659.

Xia, Y. and Hao, H. (2003). "Statistical damage identification of structures with frequency changes", Journal of Sound and Vibration, Vol. 263, No. 4, pp 853-870.

Zang, C. and Imregun, M. (2001a). "Combined neural network and reduced FRF techniques for slight damage detection using measured response data", Archive of Applied Mechanics (Ingenieur Archiv), Vol. 71, No. 8, pp 525-536.

Zang, C. and Imregun, M. (2001b). "Structural damage detection using artificial neural networks and measured FRF data reduced via principal component projection", Journal of Sound and Vibration, Vol. 242, No. 5, pp 813-827.

Zapico, J. L., Worden, K. and Molina, F. J. (2001) "Vibration based damage assessment in steel frames using neural networks", Smart Materials and Structures, Vol. 10, No. 3, pp 553-559.

Zhou, Z.H., Wu, J. and Tang, W. (2002). "Ensembling neural networks: Many could be better than all", Artificial Intelligence, Vol. 137, No. 1-2, pp 239-263. 


\section{Figure 1}

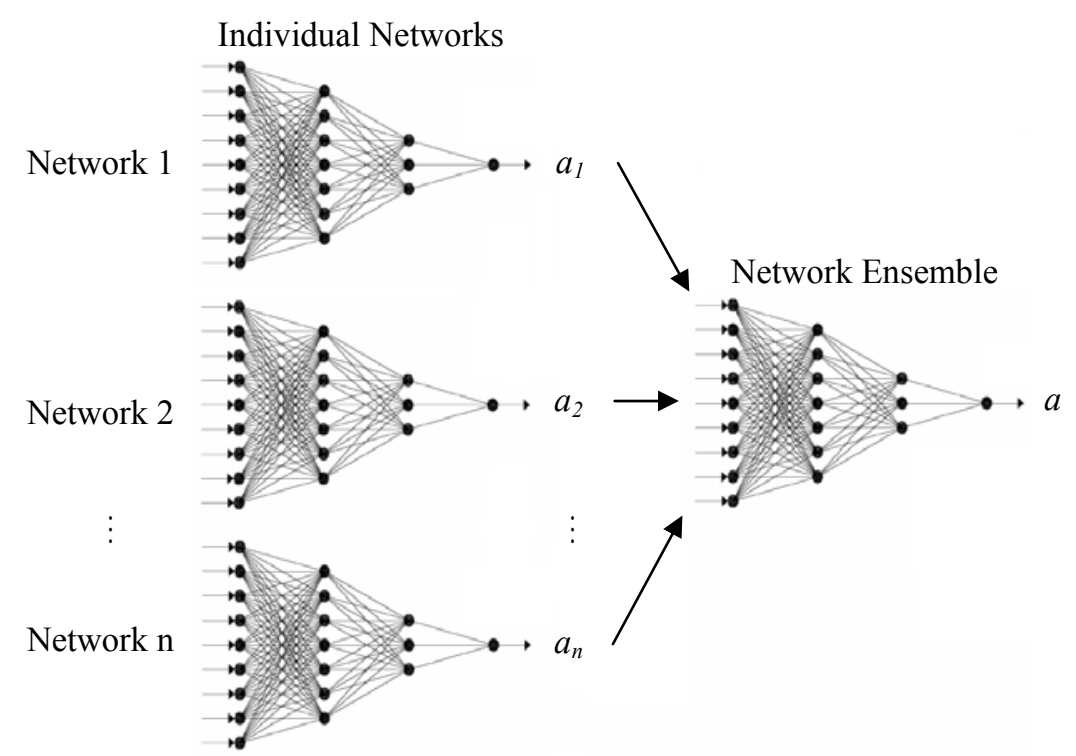

Figure 1. Model of a two-stage neural network ensemble. 


\section{Figure 2}

$\begin{array}{cccccc}\text { (1) } & 2 & 3 & 4 & 5 & 6\end{array}$

(a)

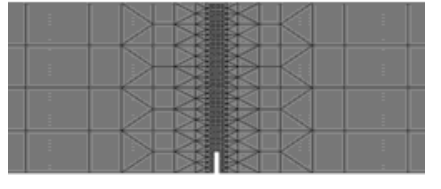

(b)

Figure 2. Finite element modelling of (a) pin-pin supported steel beam and (b) light size damage with a width of $1 \mathrm{~mm}$ and a height of $4 \mathrm{~mm}$. 


\section{Figure 3}
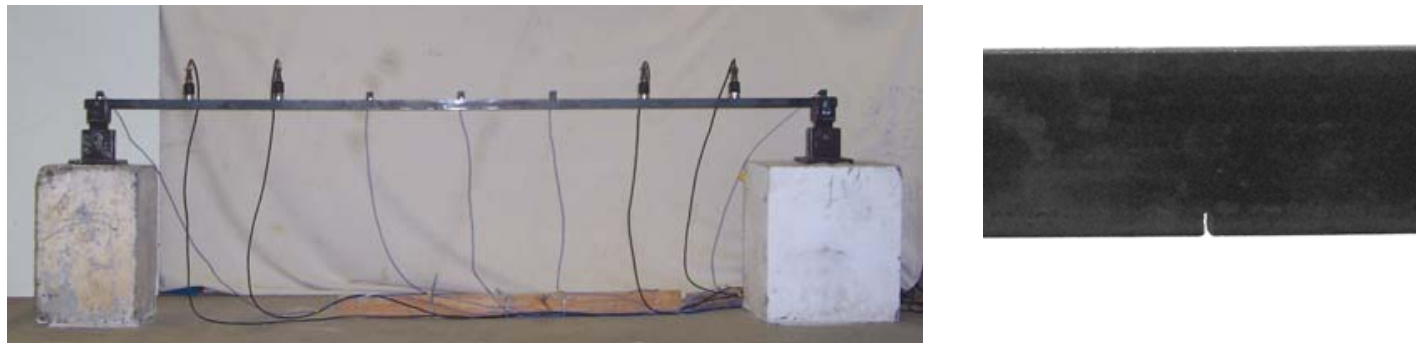

(a)

(b)

Figure 3. (a) Laboratory test set up and (b) experimental saw cut damage of light size. 


\section{Figure 4}

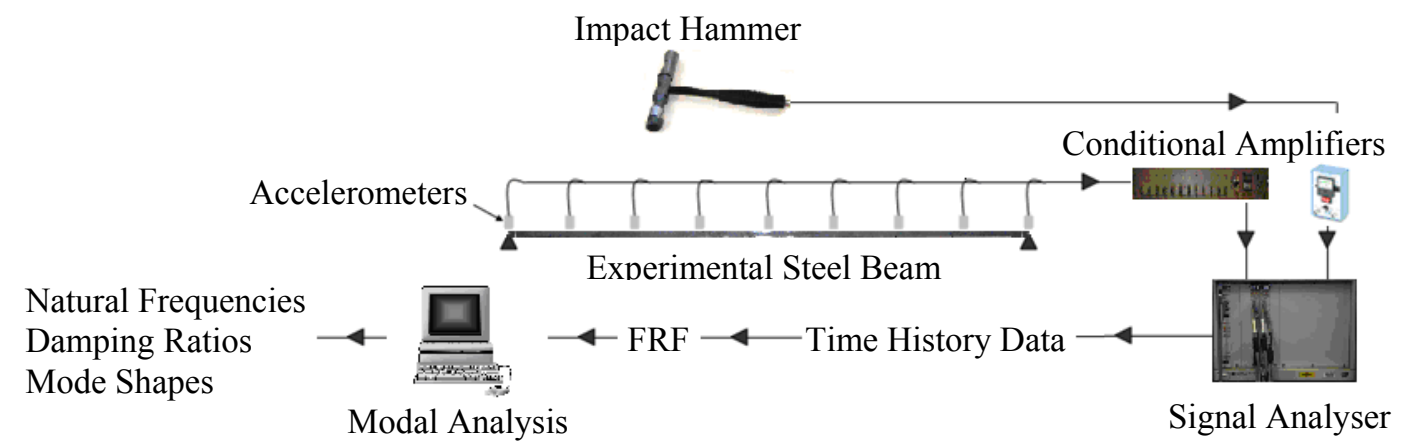

Figure 4. Schematic diagram of experimental modal testing and analysis. 


\section{Figure 5}

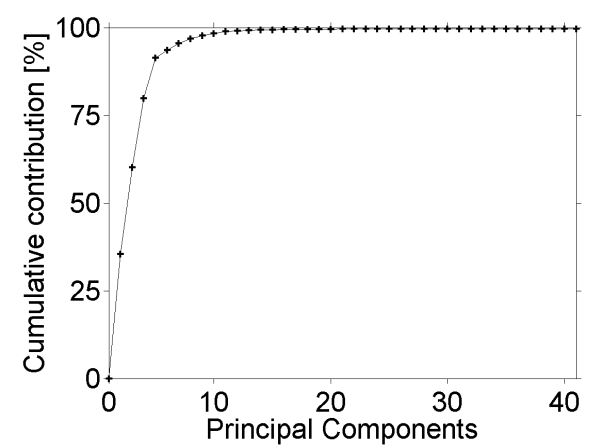

Figure 5. Cumulative contribution of PCs obtained from $Z_{j}$ derived from mode 1 of numerical data. 


\section{Figure 6}

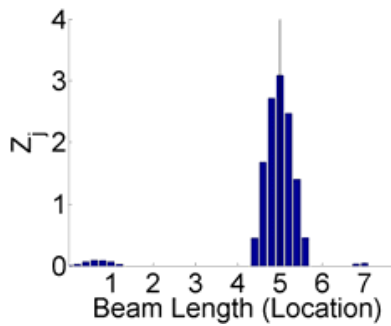

(a)

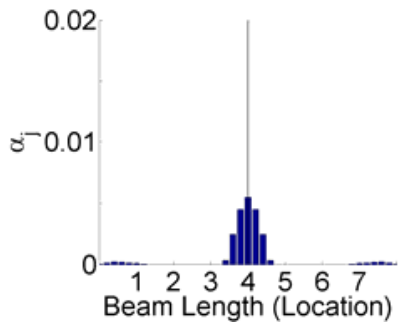

(b)

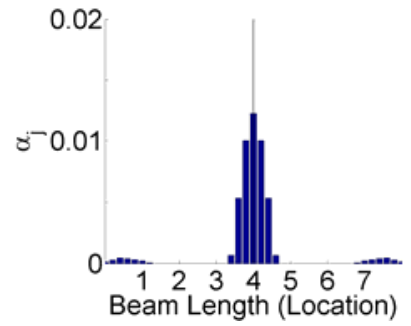

(c)

Figure 6. Damage indicators of noise-free numerical simulations derived from mode 1. (a) $Z_{j}$ of a damage situated at location ' 5 '. (b) and (c) $\alpha_{\mathrm{j}}$ of a damage situated at location ' 4 ' of medium and severe severity, respectively. 


\section{Figure 7}

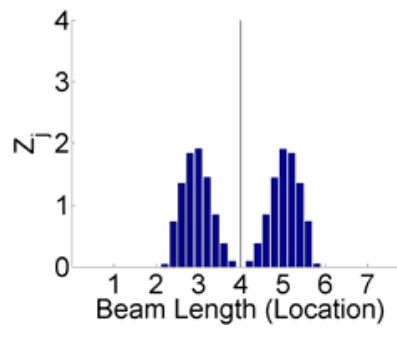

(a)

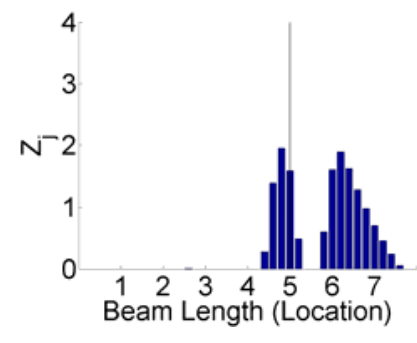

(b)

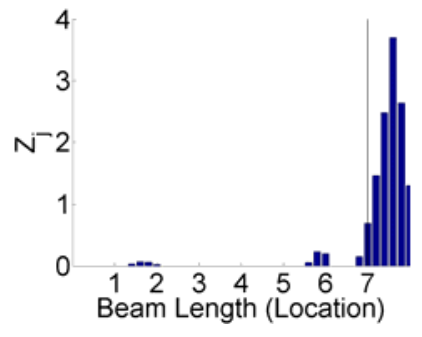

(c)

Figure 7. $Z_{\mathrm{j}}$ values of noise-free numerical simulations derived from (a) mode 4 of a beam damaged at location ' 4 ', (b) mode 3 of a beam damaged at location ' 5 ' and (c) mode 1 of a beam damaged at location ' 7 '. 


\section{Figure 8}

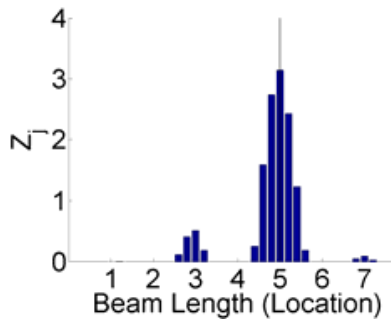

(a)

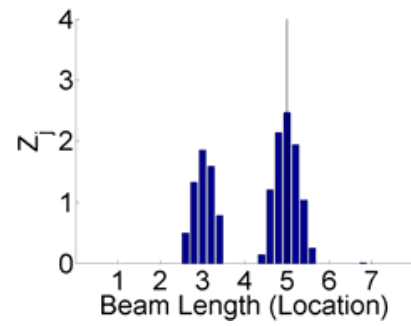

(b)

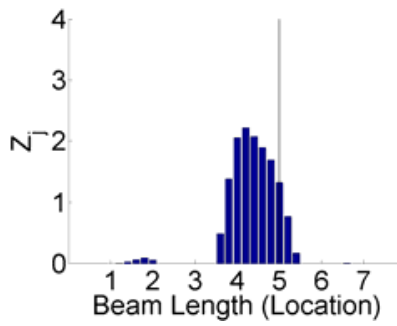

(c)

Figure 8. $Z_{j}$ values of numerical simulations polluted with three different signals of $2 \%$ white Gaussian noise derived from mode 1 of a beam damaged at location ' 5 '. 


\section{Figure 9}

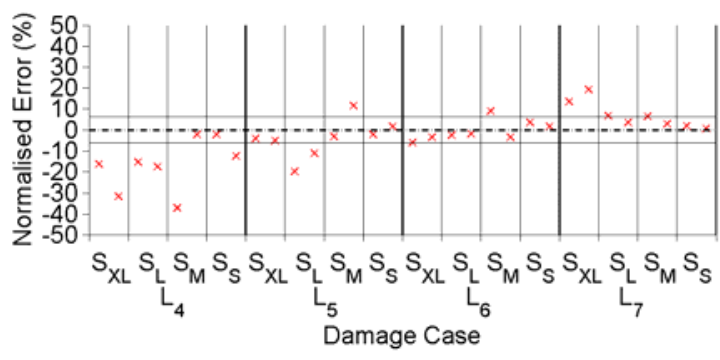

(a) mode 1 network - $1 \%$ noise

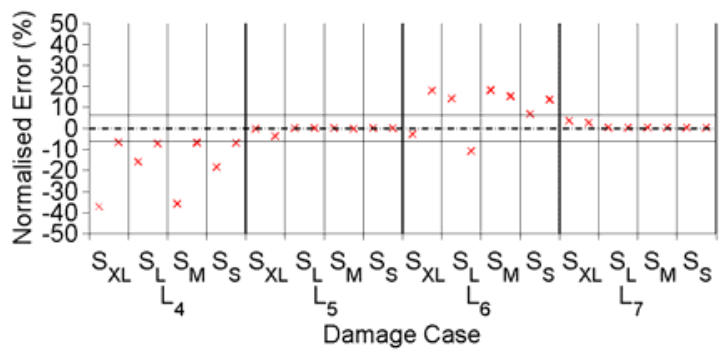

(c) mode 4 network - 1\% noise

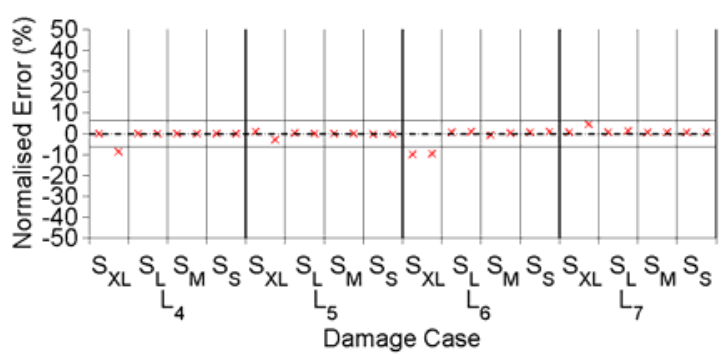

(e) mode 5 network - $1 \%$ noise

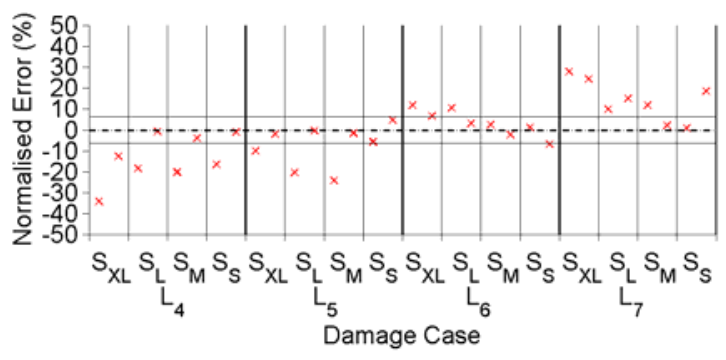

(b) mode 1 network - $10 \%$ noise

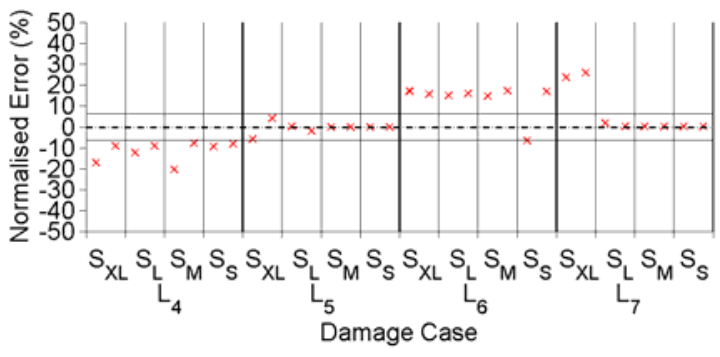

(d) mode 4 network - $10 \%$ noise

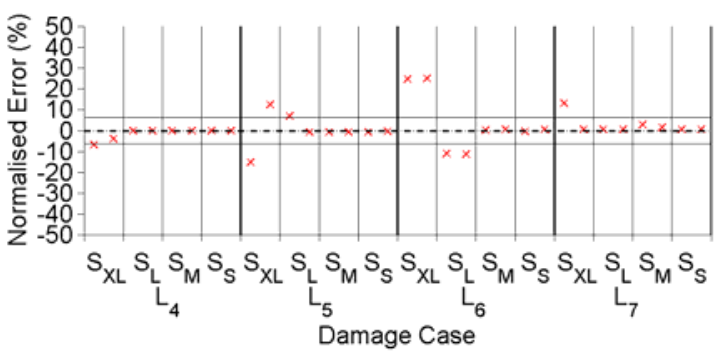

(f) mode 5 network - $10 \%$ noise

Figure 9. Testing set outcomes of individual neural networks trained with PCA-compressed $Z_{j}$ values from numerical simulations polluted with $1 \%$ and $10 \%$ white Gaussian noise, respectively, derived from (a) and (b) mode 1, (c) and (d) mode 4, and (e) and (f) mode 5. 


\section{Figure 10}

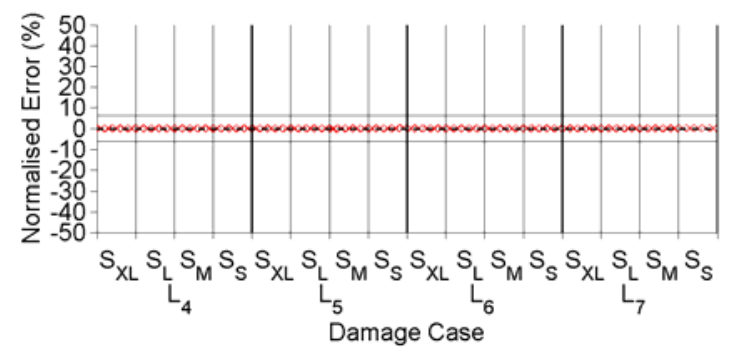

(a) damage localisation - mode 2 network

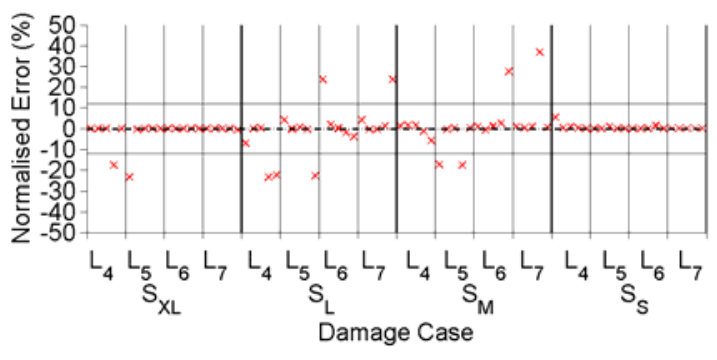

(b) damage quantification- mode 2 network

Figure 10. Testing set outcomes of individual neural networks trained with PCs of DI values derived from mode 2 of experimental beam data to (a) locate and (b) quantify damage. 


\section{Figure 11}

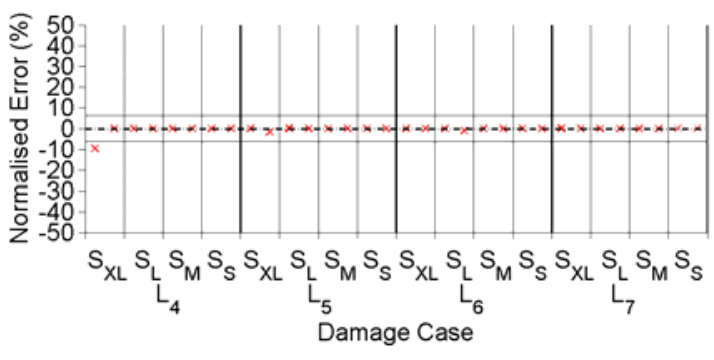

(a) network ensemble - $1 \%$ noise

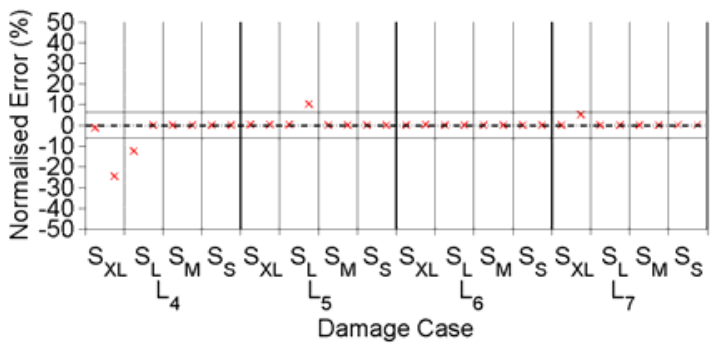

(c) network ensemble - $5 \%$ noise

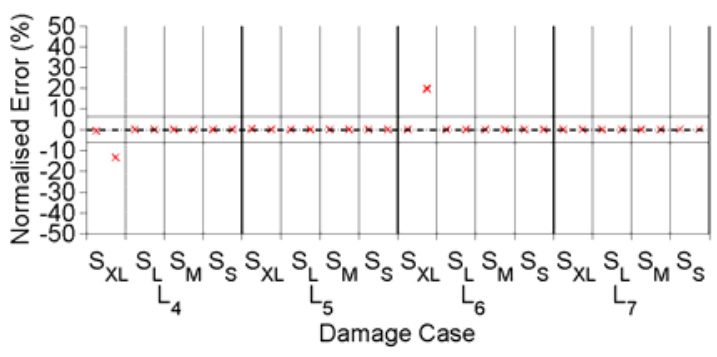

(b) network ensemble $-2 \%$ noise

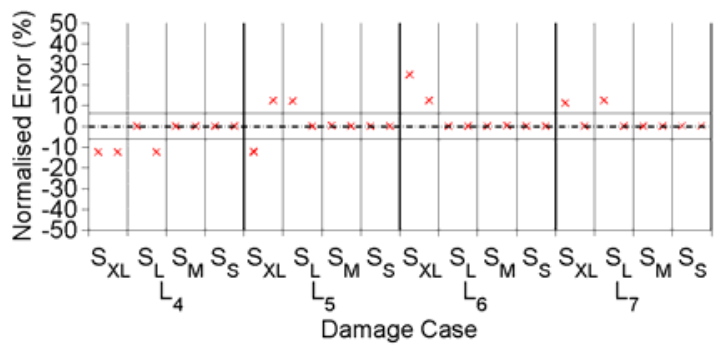

(d) network ensemble - $10 \%$ noise

Figure 11. Testing set outcomes of neural network ensemble trained with numerical data of (a) $1 \%$, (b) $2 \%$, (c) $5 \%$ and (d) $10 \%$ noise pollution to estimate the location of damage. 


\section{Figure 12}

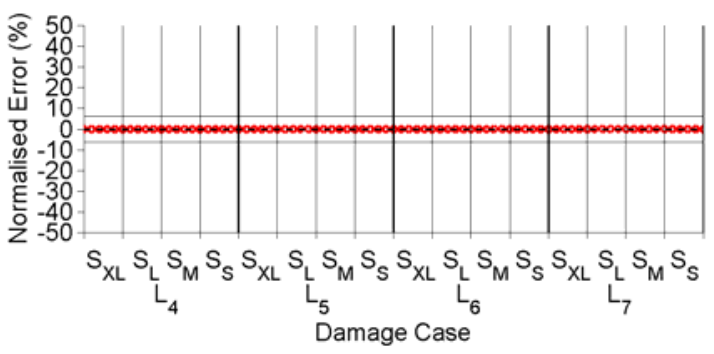

(a) network ensemble - experimental data for damage localisation

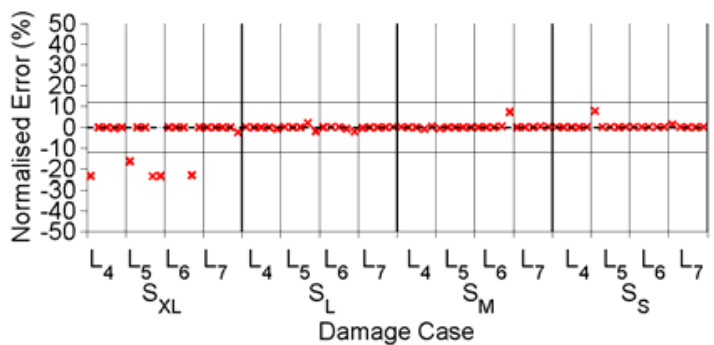

(b) network ensemble - experimental data for damage severity estimation

Figure 12. Testing set outcomes of neural network ensemble trained with experimental data to estimate (a) the location and (b) the severity of damage. 


\section{Table 1}

Table 1. Chessboard selection for laboratory beam data.

\begin{tabular}{|c|c|c|c|c|c|c|}
\hline & \multicolumn{5}{|c|}{ Undamaged data } \\
\hline & & U1 & $\mathrm{U} 2$ & U3 & U4 & U5 \\
\hline \multirow{5}{*}{$\sum_{6}^{\circ}$} & D1 & Train & Val & Train & Train & Test \\
\hline & D2 & Test & Train & Val & Train & Train \\
\hline & D3 & Train & Test & Train & Val & Train \\
\hline & D4 & Train & Train & Test & Train & Val \\
\hline & D5 & Val & Train & Train & Test & Train \\
\hline
\end{tabular}




\section{Table 2}

Table 2. Neural network performances (in absolute mean of normalised error (AMNE)) of noise-polluted numerical beam simulations to identify damage locations and damage severities.

\begin{tabular}{|l|c|c|c|c|c|c|}
\hline & \multicolumn{3}{|c|}{ Damage localisation } & \multicolumn{3}{c|}{ Damage quantification } \\
\hline Network & $\begin{array}{c}\text { Training } \\
\text { performance } \\
\text { (AMNE [\%]) }\end{array}$ & $\begin{array}{c}\text { Validation } \\
\text { performance } \\
\text { (AMNE [\%]) }\end{array}$ & $\begin{array}{c}\text { Testing } \\
\text { performance } \\
\text { (AMNE [\%]) }\end{array}$ & $\begin{array}{c}\text { Training } \\
\text { performance } \\
\text { (AMNE [\%]) }\end{array}$ & $\begin{array}{c}\text { Validation } \\
\text { performance } \\
\text { (AMNE [\%]) }\end{array}$ & $\begin{array}{c}\text { Testing } \\
\text { performance } \\
\text { (AMNE [\%]) }\end{array}$ \\
\hline Mode 1 & 8.05 & 9.29 & 10.03 & 15.47 & 18.09 & 17.61 \\
\hline Mode 2 & 4.10 & 6.52 & 6.63 & 10.13 & 11.22 & 11.76 \\
\hline Mode 3 & 3.71 & 5.27 & 5.15 & 1.40 & 4.07 & 5.12 \\
\hline Mode 4 & 3.85 & 6.68 & 6.63 & 12.32 & 14.17 & 13.30 \\
\hline Mode 5 & 1.62 & 2.96 & 2.84 & 0.20 & 0.22 & 0.52 \\
\hline Mode 6 & 3.99 & 5.48 & 4.91 & 8.89 & 9.94 & 8.55 \\
\hline Mode 7 & 1.70 & 3.94 & 2.75 & 0.03 & 1.79 & 2.05 \\
\hline Ens & 1.08 & 2.42 & 1.89 & 0.13 & 0.21 & 0.44 \\
\hline
\end{tabular}




\section{Table 3}

Table 3. Neural network performances (in absolute mean of normalised error (AMNE)) of experimental beams to identify damage locations and severities.

\begin{tabular}{|c|c|c|c|c|c|c|}
\hline & \multicolumn{3}{|c|}{ Damage localisation } & \multicolumn{3}{c|}{ Damage quantification } \\
\hline Network & $\begin{array}{c}\text { Training } \\
\text { performance } \\
\text { (AMNE [\%]) }\end{array}$ & $\begin{array}{c}\text { Validation } \\
\text { performance } \\
\text { (AMNE [\%]) }\end{array}$ & $\begin{array}{c}\text { Testing } \\
\text { performance } \\
\text { (AMNE [\%]) }\end{array}$ & $\begin{array}{c}\text { Training } \\
\text { performance } \\
\text { (AMNE [\%]) }\end{array}$ & $\begin{array}{c}\text { Validation } \\
\text { performance } \\
\text { (AMNE [\%]) }\end{array}$ & $\begin{array}{c}\text { Testing } \\
\text { performance } \\
\text { (AMNE [\%]) }\end{array}$ \\
\hline Mode 1 & 0.01 & 0.04 & 0.04 & 4.04 & 7.20 & 7.25 \\
\hline Mode 2 & 0.11 & 0.27 & 0.24 & 2.85 & 5.64 & 3.94 \\
\hline Mode 3 & 0.02 & 0.03 & 0.02 & 1.17 & 4.12 & 2.02 \\
\hline Mode 4 & 0.01 & 0.01 & 0.01 & 2.95 & 4.06 & 3.96 \\
\hline Mode 5 & 1.30 & 2.66 & 2.24 & 6.78 & 9.25 & 9.22 \\
\hline Mode 6 & 2.51 & 3.34 & 3.35 & 5.47 & 7.43 & 8.25 \\
\hline Mode 7 & 3.26 & 5.36 & 5.01 & 6.71 & 8.56 & 8.30 \\
\hline Ens & 0.01 & 0.01 & 0.01 & 1.02 & 3.21 & 1.74 \\
\hline
\end{tabular}




\section{Appendix: Notation}

$L_{\max }$ the total length of the beam

E the Elastic Young's modulus

$E_{\text {norm }}$ the normalised error of either the localisation or the quantification outcomes

I the moment of inertia of section

$Z_{i j}$ the normalised damage indicator in standard normal space

$s_{j} \quad$ the standard derivation of the $j^{\text {th }}$ column $\mathrm{x}_{\mathrm{j}}$

$S_{\max }$ the maximum severity of a damage

$\bar{x}_{j} \quad$ the mean of the $j^{\text {th }}$ column $\mathrm{x}_{\mathrm{j}}$

$\tilde{x}_{i j}$ statistically normalised $\mathrm{x}_{\mathrm{ij}}$

$\alpha_{i j}$ the damage severity estimator

$\beta_{i j}$ the damage index

$\lambda_{i} \quad$ the $i^{\text {th }}$ eigenvalue

$\left\{P_{i}\right\}$ the corresponding eigenvector of $\lambda_{i}$

$\phi_{i} \quad$ ith mode shape

$\phi_{i}{ }^{\prime \prime} \quad$ the second derivative of mode shape 